\section{THE ESTIMATION OF PHENOL IN CRUDE CARBOLIC ACID AND IN COAL-TAR OILS}

By F. W. SkIRrow

Received September 5, 1917

Until quite recently no satisfactory method was to be found in the literature for the estimation of phenol in tar oils or in crude carbolic acid. In a recent paper, Masse and Leroux ${ }^{1}$ give a method based on preliminary fractionation of the crude carbolic acid and subsequent estimation of the phenol from determinations of the solidifying point. This method suffers from the disadvantage that the cresols and other higher boiling bodies may vary in the fraction containing the phenol, while they assume a constant and arbitrary ratio of the cresols to be present.

More recently Weiss and Downs ${ }^{2}$ have published a method in which the crude carbolic acid is fractionated in such a way as to obtain the whole of the phenol in a fraction supposed to contain only phenol and the cresols, and in this fraction the phenol is then estimated by simultaneous determination of the specific gravity and the solidifying point, whence by reference to graphs the amount of phenol present is deduced. These graphs also are drawn up with fixed ratios of meta- and para-cresol.

In I909 the author worked out a method for the estimation of phenol in coal tar which depended on quite other properties of the constituents for their estimation, and which gave a fair indication of the amount of the cresols also present. The method gave satisfactory results and was used in the author's laboratory for some years, but was not at that time published. It is thought that the method of attack may not be without interest at the present time, and the author desires to thank the Directors of Messrs. Hardman and Holden, Ltd., of Manchester, England, for permission to publish the results.

In a paper on the determination of phenols in gas liquor, ${ }^{3}$ the author determined the "Oxygen $\mathrm{Ab}$ sorption" due to the various constituents of the effuent from a sulfate of ammonia works and incidentally of the phenol and cresols, respectively, in that effluent. This suggested the possibility that the oxygen absorption might furnish a means to estimate the amount of phenol in a mixture of phenolic bodies.

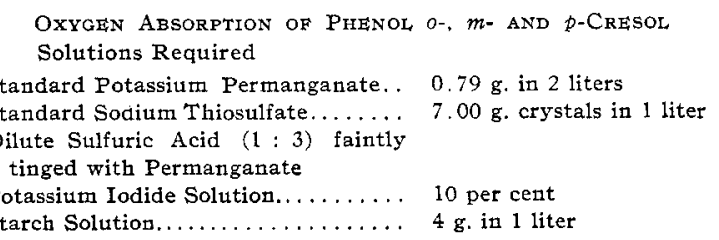

The phenol, etc., was diluted so that $50 \mathrm{cc}$. of the solution contained $0.002 \mathrm{~g}$. of the particular phenol or cresol.

\section{METHOD}

Portions ( $50 \mathrm{cc}$ ) of the permanganate were measured. .nto stoppered bottles and $\mathrm{I}_{5} \mathrm{cc}$. of the dilute sulfuric acid added to each. These bottles were placed in a rack

1 Compt. rend., 165 (1916), 361.

2 This Jovrnal. 9 (1917), 569.

J. Soc. Chem. Ind., January, 1908. in a thermostat which was maintained at $23^{\circ} \mathrm{C}$. The solutions of the tar acids were also brought to the temperature of the thermostat: $50 \mathrm{cc}$. of the tar acid solution were then run into the permanganate solution from a pipette also warmed to $23^{\circ} \mathrm{C}$, and the time noted when the first drop entered the permanganate. When the whole had been added, the bottle was quickly shaken to insure uniformity. At the end of $3 \mathrm{~min}$. the action was stopped by quickly adding I cc. of the potassium iodide solution. The liberated iodine was then titrated with the thiosulfate solution. Sufficient excess of permanganate was used so that 30 to 40 per cent of the permanganate remained at the end. The results were then calculated to grams of oxygen absorbed by $I \mathrm{~g}$. of the respective tar acid. The concordance of the results is shown in the case of the phenol, the others being equally good.

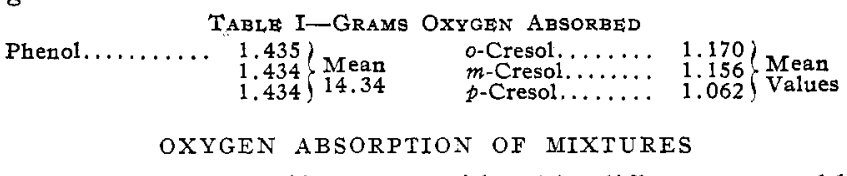

In order to see if any considerable difference would be found in the rate of oxidation of phenol if cresols were simultaneously undergoing oxidation, known mixtures were made up containing varying proportions of phenol to cresols, the cresol taken being a mixture of equal proportions of ortho, meta and para.

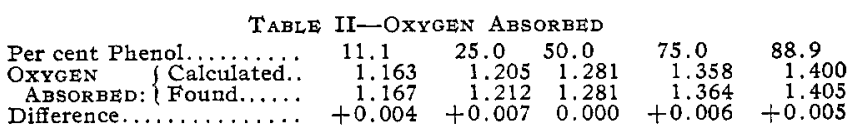

There appears to be slightly increased oxidation in most of the cases, but this is not sufficient to cause serious error in the use of the oxygen absorptions.

The rate is naturally dependent on the temperature and it is necessary to work in a thermostat. Attempts were made to work with solutions ten times as concentrated as the solutions quoled, but the results were much less steady owing no doubt partly to the considerable temperature disturbance due to the increased heat of reaction.

The phenol can thus be estimated with reasonable accuracy provided that the ratio of the cresols to one another be known. The difference in the rate of oxidation of the three cresols from one another, while much less than the difference between the rate of oxidation of any one cresol and the rate of oxidation of phenol, is still much too great to allow of estimation in this way unless the ratio of the cresols present is known. It is thus necessary to get some idea as to the ratio of cresols present in order to proceed.

\section{FRACTIONATION EXPERIMENTS}

It is obvious that in dealing with a mixture of phenol, cresols and higher boiling phenolic bodies, which are present in a crude carbolic acid, preliminary fractionation would have to precede any attempt at titration, and experiments were carried out in this direction on crude carbolic acid, which was the average from a very large amount of tar from widely differing sources. This crude carbolic acid was fractionated 
through still heads of various types and the progress of the fractionation observed by taking the oxygen absorption of the fractions.

OXYGEN ABSORPTION OF HIGHER BOILING BODIES

A large sample of higher boiling bodies was obtained which had had most of the phenol and cresols removed in a works column still. This was freed from $\mathrm{H}_{2} \mathrm{~S}$ and was fractionated several times in the laboratory to remove any phenol and cresols which might still remain. Fractions were then collected and their oxygen absorption measured with the results given in Table III

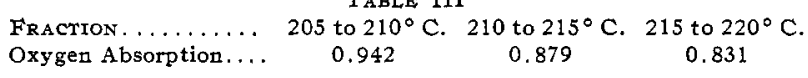

The oxygen absorption decreases steadily with increasing boiling point and will thus throw light on the progress of the fractionation.

\section{COMPARISON OF THE EFFICIENCY OF STILL HEADS}

The two still heads giving the best results with crude carbolic acid were found to be the "LeBel" bulbs and the "Pear" bulbs. The Hempel column was not so efficient and had the disadvantage that a large amount of liquid is held up in the glass beads. The Young dephlegmator, although excellent for lower boiling liquids, did not give as good results in the author's hands as did the Pear bulbs.

COMPARISON OF A 4-BULB LEBEI WITH A I2-BULB PEAR STILL HEAD-The total length of the two columns to the side tube was practically the same. In both cases I000 cc. of the crude carbolic acid was taken in a copper flask and distilled through the respective head. An air condenser was used leading into receivers closed with a calcium chloride tube. Any moisture was first distilled off and the temperature carried to $180^{\circ} \mathrm{C}$. The phenol was recovered from this, dehydrated, and returned to the distilling flask and the fractionation then proceeded with. The rate of distillation was regulated carefully to one d:op per second, a seconds pendulum being placed behind the receiver. The flask was protected by a shield of asbestos paper and the column enclosed in one thickness of glazed paper to prevent draughts and to diminish somewhat the radiation from the column. Under these conditions extremely steady conditions were easily maintained. A small Anschütz thermometer graduated in tenths was used and this was compared with a standard "N. P. L." thermometer.

The oxygen absorption of each fraction was measured and the fractions then submitted to a second and to a third systematic fractionation. The oxygen absorption of the fractions of the second and of the third fractionations were also measured. Table IV contains the results so obtained. The two first temperature intervals in the first fractionation are different from those in the second and third fractionations, but in all subsequent work the latter intervals were used.

If we examine these fractionations we see that the oxygen absorption of the fractions is much the same whether the "Pear" or the "LeBel" column is used, being a little to the advantage of the "Pear" form but that with the Pear a greater volume accumulates in the first fraction which contains the largest proportion of phenol. The use of the Pear head is further to be preferred on account of the smaller amount of liquid remaining after a distillation. In all subsequent work the Pear form was used. This superiority of the Pear still head was also established by Weiss and Downs. ${ }^{1}$

It will further be seen by comparing the second and the third fractionations, confining our attentions now to the values for the Pear head only, that the "quality" of the fractions does not alter very materially after the second fractionation, but that the chief difference is in the volume of the first fraction. From this it was inferred that more than two fractions would not bring any commensurate advantage, and that with two fractionations a steady state of affairs had been reached which would be easily reproducible. The examination of these fractions was then proceeded with, to ascertain the amount of phenol in each.

The oxygen absorption of the fractions obviously gives us no quantitative measure of the amount of phenol which they contain as we have no information as to the ratio in which the cresols exist in each.

\section{MATCHING EXPERIMENTS}

In order to establish definitely the percentage of phenol, ortho-, meta- and para-cresol in these fractions, use was made of a fact previously observed by the author in the course of work on the phenols in gas liquor but not at that time alluded to, viz., that if the Messinger and Vortman method for the estimation of phenol be applied to solutions of $o-, m$ - and $p$-cresol, respectively, the end-product obtained (triiodophenol, etc.) has widely different colors in each case. The colors of these end-products are extremely characteristic and may be described as follows:

$\begin{array}{cccc}\text { PhenOL } & \text { O-Cresor } & \text { m-Cresol } & \text { p-CRESOL } \\ \text { Bright Rose-Pink } & \text { Brown } & \text { Slate-Blue } & \text { Dirty Yellow }\end{array}$

Preliminary experiments showed that if a known mixture of phenol and the cresols be taken, the percentage of each constituent could be determined with good accuracy by first measuring the oxygen absorption of the mixture and then making up mixtures with the same oxygen absorption, forming the iodo-compounds, and by the method of trial and failure gradually approaching the exact shade of the so-called tri-iodocompound of the mixture to be analyzed. (The endproduct in the case of the cresols is by no means exactly the tri-iodo compounds.)

The iodo compound was prepared in each case as follows: $200 \mathrm{cc}$. of water and $3.5 \mathrm{cc} . N \mathrm{NaOH}$ were mixed; I $\mathrm{g}$. of the tar acid was dissolved in $500 \mathrm{cc}$. of water and $25 \mathrm{cc}$. of this solution were added to the dilute sodium hydroxide solution. This was heated to $70^{\circ} \mathrm{C}$. in a stoppered flask and $25 \mathrm{cc}$. of $0.02 \mathrm{~N}$ iodine solution added and well shaken. After standing for 5 mins. the flask was cooled under the tap, the mixture slightly acidified with sulfuric acid and the excess of iodine removed with a slight excess of sodium

1 Loc. cit. 


\begin{tabular}{|c|c|c|c|c|c|c|c|c|c|c|c|c|c|c|c|}
\hline \multirow{9}{*}{$\begin{array}{c}\text { No. } \\
1 \\
2 \\
3 \\
4 \\
4 \\
5 \\
6 \\
7 \\
8 \\
8 \\
9 .\end{array}$} & \multicolumn{2}{|c|}{ (1)-FIRST FrRACTIONAT } & $\begin{array}{l}\text { TABLE IV-C } \\
\text { ON }\end{array}$ & ARISO & $\begin{array}{r}\text { on of 4-BUL } \\
(2)-\mathrm{SE}\end{array}$ & $\begin{array}{l}\text { LEB } \\
\text { OND }\end{array}$ & $\begin{array}{l}\text { WI WITE } \\
\text { RACTIO }\end{array}$ & A $12-B$ & $30 L B P E$ & STII & $\begin{array}{l}\text { FEEAD } \\
(3)-T\end{array}$ & & & & \\
\hline & peratu & Vol. o & vol. & & mpe & Vol & 0 & Vol. & & & mperatu & Vo & & $\mathrm{Co}$ & \\
\hline & & C & Cc. & No. & & & & & & No. & & & & Cc. & \\
\hline & $\begin{array}{l}\text { To } 185.5 \\
185.5 \text { to } 187\end{array}$ & $\begin{array}{rl}26 & 1.293 \\
155 & 1.305\end{array}$ & $\begin{array}{rr}27 & 1.29 \\
112 & 1.30\end{array}$ & $\frac{1}{2}$ & $\begin{array}{l}18 \\
18\end{array}$ & $\begin{array}{l}143 \\
179\end{array}$ & $\begin{array}{l}1.3 \\
1.2\end{array}$ & 12 & & 1 & 184 & 202 & & 165 & \\
\hline & $\begin{array}{l}0190 \\
0 \\
0\end{array}$ & $\begin{array}{l}234 \\
103\end{array}$ & $\begin{array}{l}243 \\
130\end{array}$ & $\overline{3}$ & 18 & 75 & 1.2 & 114 & & $\begin{array}{l}2 \\
3\end{array}$ & $\begin{array}{l}18 \\
18\end{array}$ & $\begin{array}{r}100 \\
87\end{array}$ & & $\begin{array}{r}126 \\
90\end{array}$ & \\
\hline & 0 & 69 & 811.1 & 5 & & 65 & 1.1 & 66.5 & & 4 & 190 to 1 & 6 & & 64 & 1. \\
\hline & $\begin{array}{l}\text { to } 199 \\
\text { to } 202\end{array}$ & $\begin{array}{l}61 \\
67\end{array}$ & $\begin{array}{l}62 \\
69\end{array}$ & ${ }_{7}^{6}$ & & $\begin{array}{l}66 \\
61\end{array}$ & $\begin{array}{l}1.0 \\
1.0\end{array}-50$ & $\begin{array}{l}69 \\
54\end{array}$ & 1. & 5 & 19 & 3 & 1. & 66 & 1. \\
\hline & 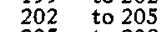 & r & 7 & & & & & & & 6 & & 85 & & 67 & 1.094 \\
\hline & & & & & & 23 & & i) & & 7 & 199 to 202 & 71 & 1.060 & 71 & 1.058 \\
\hline
\end{tabular}

thiosulfate solution. The precipitated iodo compound was allowed to settle, washed once by decantation and placed in a small clear glass weighing bottle for comparison. It is essential that the compound be freshly prepared as it becomes lighter in color after standing one or two hours. The method is clearly cumbersome but it was used only to establish the percentages present in the fractions once and for all, $i$. e., as a method of standardization.

In this way the percentages of phenol, $0-, m$-, and $p$-cresol were determined in the fractions through the Pear still head for the second and third fractionations quoted in Table IV, (2) and (3).

\begin{tabular}{|c|c|c|c|c|c|c|c|c|c|c|c|}
\hline \multirow{2}{*}{\multicolumn{6}{|c|}{ 1-SECOND FRACTIONATION }} & \multicolumn{6}{|c|}{ 2-THIRD Fractionation } \\
\hline & & & & & & 0 & Vo & $\overparen{P h e}$ & & Cresal & \\
\hline No & 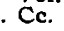 & 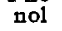 & 0 & $p$ & $m$ & Abs. & $\mathrm{Cc}$ & no. & 0 & $p$ & $m$ \\
\hline $\begin{array}{l}1 \\
2 \\
3 \\
4 \\
5 \\
6 \\
7\end{array}$ & $\begin{array}{r}143 \\
179 \\
75 \\
78 \\
65 \\
66 \\
61\end{array}$ & $\begin{array}{r}71.0 \\
56.0 \\
40.5 \\
30.0 \\
15.9 \\
6.9 \\
0\end{array}$ & $\begin{array}{l}14.8 \\
20.8 \\
18.7 \\
22.2 \\
18.7 \\
18.6 \\
11.3\end{array}$ & $\begin{array}{l}14.2 \\
23.2 \\
40.8 \\
47.8 \\
65.4 \\
65.7 \\
72.8\end{array}$ & $\begin{array}{c}0 \\
0 \\
0 \\
0 \\
0 \\
8.8 \\
15.9\end{array}$ & $\begin{array}{l}1.117 \\
1.114 \\
1.096 \\
1.083 \\
1.076 \\
1.073 \\
1.068\end{array}$ & $\begin{array}{r}202 \\
100 \\
87 \\
56 \\
53 \\
85 \\
70\end{array}$ & $\begin{array}{r}73.5 \\
57.1 \\
40.5 \\
25.0 \\
15.4 \\
3.2 \\
0\end{array}$ & $\begin{array}{l}14.5 \\
21.4 \\
25.5 \\
25.0 \\
21.1 \\
19.8 \\
11.3\end{array}$ & $\begin{array}{l}12.0 \\
21.4 \\
34.0 \\
50.0 \\
63.5 \\
68.5 \\
72.8\end{array}$ & $\begin{array}{r}0 \\
0 \\
0 \\
0 \\
0 \\
9 . \\
15 .\end{array}$ \\
\hline
\end{tabular}

The mixtures were made up to have the same oxygen absorptions as the respective fractions with the exception of the higher fractions-where it was found that better matches could be obtained by making mixtures with slightly higher oxygen absorptions than the fractions. This was probably due to the small amounts of higher boiling bodies in the higher fractions. (The color of the "iodo-compounds" of the higher boiling bodies is not pronounced, while their oxygen absorptions are progressively less than the cresols.) Table $\mathrm{V}$ gives the results of these matching experiments. The last column gives the mean oxygen absorption of everything except phenol in the fractions, calculated from the oxygen absorption of the fraction, the oxygen absorption of pure phenol and the found percentage of phenol in the fraction. Here we see more definitely that the percentage of phenol in the third fractionation has not changed very materially from that in the second fractionation.

\section{RESULTS OBTAINED WITH OTHER MATERIALS}

The preceding results were obtained with a large stock sample of crude carbolic acid representing the total extract from a very large amount of tar. Similar experiments were then carried out with other materials as follows:

\section{A-For comparison: Material already quoted \\ B-Sample of Refined Cresylic Acid \\ C-Sample of " 60 's" Carbolic Acid \\ D-Another sample of Refined Cresylic Acid of different origin \\ E-Another sample of Crude Carbolic Acid of different origin to that quoted in " $A$ "}

These were submitted to preliminary purification to free them from $\mathrm{H}_{2} \mathrm{~S}$, etc., and were then fractionated twice in the manner described. In $A$ the volume of the fractions was measured; in all subsequent operations the fractions were weighed to the nearest decigram. Results are given in Table VI.

In Table VII is given the mean oxygen absorption of everything except the phenol in the fractions, calculated as before indicated from the oxygen absorption of the fraction, the oxygen absorption of pure phenol and the found percentage of phenol in the fraction. From these data we see that the mean oxygen absorption of the cresols, etc., in the fractions is very constant for one and the same fraction and varies very little with the nature of the material distilled. Thus. it seems evident that the larger variation of the oxygen absorption of the fractions themselves is caused principally by variation in the percentage of phenol present.

\section{METHOD OF ANAIYSIS ADOPTED}

Thus the method adopted for the analysis of any unknown sample of crude carbolic acid was to fractionate about a liter of the sample in the manner outlined and then determine the oxygen absorptions of the fractions of the second fractionation. The per-

TABLE VI-RESULTS WITH VARIOUS MATERIAIS

\begin{tabular}{|c|c|c|c|c|c|c|}
\hline No. & $\begin{array}{l}\text { Temp. } \\
\text { c. }\end{array}$ & $\begin{array}{c}\mathrm{AM} \\
\mathrm{A} \\
\mathrm{Cc} .\end{array}$ & B. & $\begin{array}{c}\text { C. } \\
\text { G. }\end{array}$ & D. & $\stackrel{\mathrm{E}}{\mathrm{G}}$ \\
\hline $\begin{array}{l}1 \\
2 \\
3 \\
4 \\
5 \\
6 \\
7\end{array}$ & $\begin{array}{l}180 \text { to } 184 \\
184 \text { to } 187 \\
187 \text { to } 190 \\
190 \text { to } 193 \\
193 \text { to } 196 \\
196 \text { to } 199 \\
199 \text { to } 202\end{array}$ & $\begin{array}{r}143 \\
179 \\
75 \\
78 \\
65 \\
66 \\
61\end{array}$ & $\begin{array}{l}41.6 \\
173.8 \\
200.6 \\
115.5 \\
109.6 \\
104.2 \\
106.1\end{array}$ & $\begin{array}{r}377.3 \\
152.1 \\
68.7 \\
45.1 \\
37.7 \\
23.5 \\
22.6\end{array}$ & $\begin{array}{r}7.4 \\
58.3 \\
171.2 \\
151.1 \\
128.3 \\
125.5 \\
147.4\end{array}$ & $\begin{array}{r}44 . \\
183 . \\
104 . \\
76 . \\
70 . \\
56 . \\
63 .\end{array}$ \\
\hline
\end{tabular}

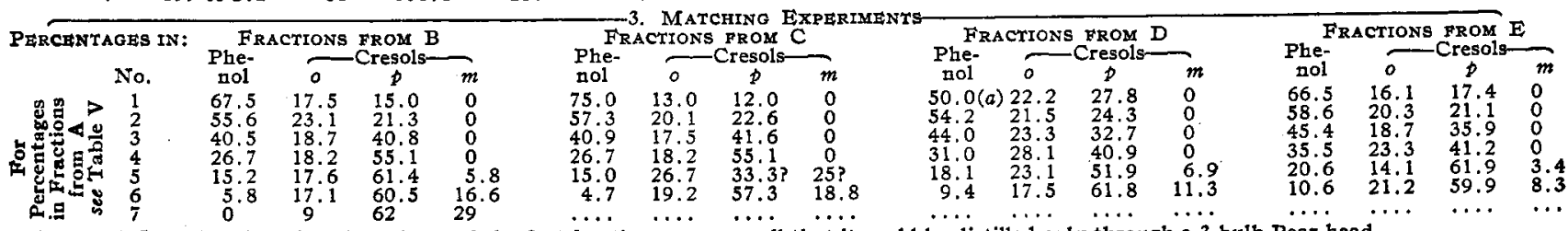

(a) On thejfirst fractionation the volume of the first fraction was so small that it could be distilled only through a 3 -bulb Pear-head.

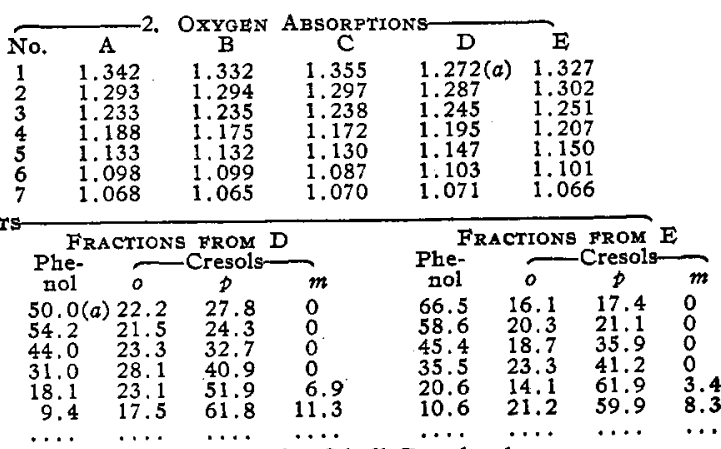


TABLE VII-MEAN OXYGEN ABsorption OF ThE CRESOLS, ETC.

\begin{tabular}{|c|c|c|c|c|c|c|}
\hline & & & & & & \\
\hline & A & & C & 0 & $\mathbf{E}$ & \\
\hline & $\begin{array}{l}1 . \\
1.0 \\
1.0 \\
1.0 \\
1.0\end{array}$ & $\begin{array}{l}1 . \\
1 . \\
1 . \\
1.0 \\
1.0 \\
1 .\end{array}$ & $\begin{array}{l}1 . \\
1 . \\
1 . \\
1 . \\
1 .\end{array}$ & $\begin{array}{l}0) \\
3 \\
7 \\
8 \\
5 \\
7\end{array}$ & & \\
\hline
\end{tabular}

centage of phenol in each fraction was then calculated from the oxygen absorption of pure phenol and the oxygen absorption of the cresol, etc., in that fraction as given in Table VII. In Table VIII comparison is made of the percentages of phenol found in the materials $\mathrm{A}, \mathrm{B}, \mathrm{C}, \mathrm{D}$ and $\mathrm{E}$

(I) By direct matching of the fractions.

(2) By calculation, as described, from the mean oxygen absorptions of the cresols, etc., in the respective fraction.

These results are not, of course, independent, as the results in the second case also depend on the matching experiments, but the result indicates how far the mean oxygen absorption of the cresols, etc., in the fractions may be regarded as fixed points, thus avoiding the necessity for the tedious matching experiments in each case dealt with.

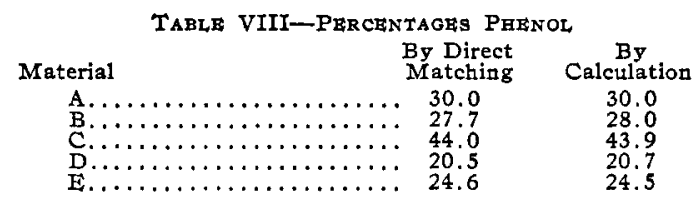

SOURCES OF ERROR IN THE PRECEDING WORK

I-It has been noted that in the case of the higher boiling fractions better matches were obtained when mixtures were made up having slightly higher oxygen absorption than the fractions to be matched, and it was pointed out that this was probably due to increasing amounts of higher boiling bodies in these fractions. It would undoubtedly have been better to have taken some of this higher boiling material as well as phenol and the cresols in the matching experiments.

2-It was found after the method had been in use for some time that in practically all experiments with crudes a disturbing factor was present in the form of small amounts of pyridine.

3-It was further observed that crude carbolic acid tenaciously retains small amounts of dissolved sodium phenate when water is also present. Thus water is much more soluble in crude-tar acids in presence of sodium phenate, and, on the other hand, sodium phenate is considerably soluble in tar acids in presence of water. This sodium phenate remains in the distilling flask when the mixture is distilled. It was found necessary to treat the crude carbolic acid with successive washes of dilute sulfuric acid, followed by washes of water to remove pyridine and decompose the dissolved sodium phenate. The tar acids in the acid and water washes were recovered and returned to the bulk.

When these disturbing factors were discovered, time was not available to repeat the work herein recorded, but the effects were partially eliminated by using the constants already determined on a series of synthetic mixtures with known amounts of phenol and varying and known amounts of the cresols and higher boiling bodies. In that way a correction was found to be applied to all results as so obtained.

The resulting corrections when plotted against the percentage of phenol found gave a smooth curve. Typical points from this curve are given in Table IX. TABLE IX

$\begin{array}{rrrrrr}\text { Per cent Phenol Taken............. } & 10.00 & 25.00 & 50.00 & 75.00 \\ \text { Found.............. } & 9.62 & 23.98 & 47.50 & 70.54\end{array}$

Correction (per cent) ............

Duplicate determinations when carried out with the precautions mentioned on the same sample of material gave results with a maximum variation of not more than about 0.2 to 0.3 per cent and with the above corrections applied were not very much farther than that from the truth.

OXYGEN ABSORPTION OF THE FRACTIONS OF TAR ACIDS FROM VARIOUS KINDS OF TAR

The method was also used to estimate the phenol in the tar acids from inclined retorts, vertical retorts, blast furnace creosote and producer gas tar. In Table $X$ are quoted the oxygen absorption of the fractions of the second fractionation of these materials. The first column contains results already given which were largely for the material from horizontal retorts. With the exception of the first column the tar acids received the preliminary treatment with dilute sulfuric acid, and were further carefully freed from any neutral oils by conversion to phenate and extraction with benzene, with all necessary precautions to recover any phenol which was so washed out.

It will be seen that these oxygen absorptions all lie in much the same range.

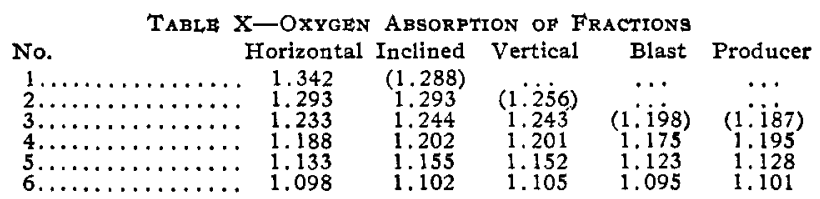

The figures given in parentheses were for very small fractions which could not be handled through the I 2 bulbs.

REMARKS

It will be noted from the matching experiments that the fraction from 199 to $202^{\circ}$ always contains definite and easily detectable percentages of phenol, but that in the fraction above $202^{\circ}$ no phenol was ever detected. Weiss and Downs also distilled twice, but collected only two fractions, viz., "to $190^{\circ}$ " and "I 90 to $202^{\circ}$ " on the first fractionation, while on the second they collected everything up to $197^{\circ}$. It seems certain that under these conditions some phenol must have been left in the distilling flask, while some of the higher boiling bodies must have inevitably appeared in the distillate.

Another point which is of interest in connection with the work of Weiss and Downs is to be found in the amount of $m$-cresol present. In the materials investigated by the author the amount of $m$-cresol in the fractions up to $202^{\circ}$ was invariably far less than the amount of 0 -cresol or of $p$-cresol. Thus from the figures in Tables IV, V and VII the following 
ratios may be calculated for the materials designated "A," "B" and "C."

$$
\begin{aligned}
& \mathrm{A}-0: p: m=6.5: \text { I } 2.8: \text { I } \\
& \mathrm{B}-0: p: m=6.0: \text { I } 3.0: \text { I } \\
& \mathrm{C}-0: p: m=8.3: \text { II. } 5: \text { I }
\end{aligned}
$$

As meta-cresol boils only $3^{\circ}$ higher than $p$-cresol, it seems certain that in the original materials dealt with the amount of $p$-cresol must much exceed the amount of $m$-cresol. Weiss and Downs in the preparation of their graphs made up synthetic mixtures in which the ratio of para to meta was fixed at about I : I and they state that that is the approximate ratio in which they exist in their oils.

The question arises whether their method would give reliable results when applied to the tar acids dealt with by the author. In the present method such variation in the ratios of the cresols present would be eliminated, at any rate to a considerable extent, although the ratio of cresols worked with in the preparations of the synthetic mixtures was not varied as far as to include a ratio, $m: p=I: x$, so that definite figures cannot be given.

It would be interesting to follow up this and the other points raised at greater length but the author has not now access to the necessary crude materials.

Chemicai DePARTMENT, MCGIIL UNIVERSity Montreal. Canada

\section{ANALYTICAL CONTROL OF THE AMMONIA OXIDATION PROCESS ${ }^{1}$}

By GUY B. TAYLOR AND JOS. D. DAvis Received October 17, 1917

In the manufacture of nitric acid from ammonia, a mixture of air and ammonia gas is passed over a suitable catalytic material heated to temperatures above red heat. Two reactions occur:

$$
\begin{aligned}
& 4 \mathrm{NH}_{3}+5 \mathrm{O}_{2}=4 \mathrm{NO}+6 \mathrm{H}_{2} \mathrm{O} \\
& 4 \mathrm{NH}_{3}+3 \mathrm{O}_{2}=2 \mathrm{~N}_{2}+6 \mathrm{H}_{2} \mathrm{O}
\end{aligned}
$$

It has been suggested that reaction (2) may come about by means of the intermediate reaction, ${ }^{2}$

$$
4 \mathrm{NH}_{3}+6 \mathrm{NO}=5 \mathrm{~N}_{2}+6 \mathrm{H}_{2} \mathrm{O} \text {. }
$$

In testing out a particular type of commercial oxidizer, the authors believe they have found evidence that under certain conditions this reaction does occur. The oxidizer in question was so constructed that the burned gases remained hot for some time. Further, there was irregular local cooling of the catalyst due to eddy currents in the burned gases. Consequently, there must have been considerable ammonia passing such points unburned. In fact, analysis of samples taken from points near the cold spots showed ammonia while in samples taken outside the catalyst chamber the ammonia content was very low. The authors believe that most of the $\mathrm{NH}_{3}$ passing through the cold spots was subsequently "burned" by the hot NO. Results with this apparatus were uniformly about ro per cent lower than those obtained with the same lot of catalytic material in a differently constructed oxidizer.

The nitric oxide is converted into nitric acid by passing the gases through suitable absorption towers,

1 Published by permission of Director of U. S. Bureau of Mines.

2 Reinders and Cats, Chem. Weekblad, 9 (1912), 47-58. much the same as in the arc process of direct fixation of atmospheric nitrogen.

The efficiency of an ammonia converter depends upon establishing conditions favorable to reaction ( $I$ ) and suppressing as far as possible reaction (2). If the ultimate product desired is nitric acid, little or no free ammonia should be allowed to pass the converter unchanged.

In order to test the efficiency of conversion, analyses of the entering ammonia-air mixture and the exit nitrose gases must be made. The former offers no special difficulties but the nature of the acid gas creates a special problem. As soon as the gas sample cools, the nitric oxide begins to react with the excess oxygen present to form $\mathrm{NO}_{2}$, which partially dissolves in the condensed water, so that the gas taken into any sampling device consists of a mixture of nitrogen, oxygen, and nitrogen oxides of indeterminate molecular species.

The principle upon which the efficiency calculation is based is as follows: the ratio of the nitrogen combined as ammonia to total nitrogen in the intake gas is equal to the ratio of the nitrogen derived from the ammonia to total nitrogen in the exit gas.

Let $a=$ nitrogen combined as $\mathrm{NH}_{3}$ in the air-ammonia

$$
b=\text { free nitrogen }
$$

$$
\left.\begin{array}{c}
d=\text { nitrogen combined as nitrogen oxides } \\
f=\text { nitrogen combined as ammonia escap- } \\
\text { ing oxidation }
\end{array}\right\} \begin{gathered}
\text { in the } \\
\text { exit gas } \\
\text { sample }
\end{gathered}
$$

The nitrogen in each sample must of course be expressed in terms of the same unit. Then

$$
\frac{a}{a+b}=\frac{c+f+x}{c+d+f}
$$

where $x$ is the free nitrogen derived from the reaction expressed by Equation (2). The efficiency is then expressed by the relation

$$
\frac{c}{c+f} \overline{+x}=\text { Yield }
$$

or, substituting the value of $x$ from (3),

$$
\frac{c(a+b)}{a(c+d+f)} \times 100=\text { Per cent Yield. }
$$

\section{EXPERIMENTAL METHODS}

In the course of some experiments on ammonia oxidation, several methods of procedure were developed:

METHOD I-The apparatus is sketched in Fig. I. Air was passed through the meter $A$, the water seal and pressure gauge $B$, and the bottles $C$ containing ammonia liquor ( 7 to 8 per cent $\mathrm{NH}_{3}$ ). The ammoniaair mixture passed over the catalyzer in $D$ and the acid gases out through $E$.

The tubes $H H^{\prime}$, capacity about $1200 \mathrm{cc}$., were filled with water. $K$ contained 20 cc. $N / 5 \mathrm{H}_{2} \mathrm{SO}_{4}$ and $K^{\prime \prime}$ I 5 cc. $N / 2 \mathrm{NaOH}$ plus 3 cc. of 3 per cent hydrogen peroxide.

The ammonia-air sample was drawn through $K$ by allowing a measured volume of water to flow from $H$. Before drawing the acid sample the air in $K^{\prime \prime}$ was displaced by pure oxygen passing in at $G$ and out at $F$. The two samples were then drawn simultaneously so 